\title{
EDITORIAL Applied models and the use of quantitative techniques
}

\begin{abstract}
From time to time there appears in the literature a survey of the use of OR techniques. Typical conclusions from these surveys are that statistical analysis and simulation are the most used techn-iques, but that other standard quantitative techniques usually included on OR courses appear to be relatively little used. The experience of many practitioners bears this out: 'We do lots of good work but we hardly ever use the techniques discussed in most OR textbooks'. However, the conclusions of such surveys need to be treated with caution since they can easily be criticised on methodological grounds. For example, surveys of identified operational researchers don't reveal what use is being made of OR techniques by other groups of people not included in the survey. Another difficulty is defining the nature of a given 'technique' and what constitutes use of the technique. In the first place it is important to distinguish between the processes of problem formulation and problem evaluation.
\end{abstract}

Problem formulation typically involves model building of some description, whether quantitative or qualitative. The classic approach is to recognise that a given situation can be represented by a general OR model with suitable modifications. In principle, evaluation is then straightforward using the body of (mathematical) methods that have been developed to evaluate the generic model form and its variations. For example, a production mixing problem might be represented by an LP model formulation. Depending on the size and particular form of the model different methods might be used to transform the model, calculate optima and produce sensitivity information. Thus the term 'technique' might be reserved for methods of model evaluation (identifying feasible solutions and optima in LP problems), but often it includes the process of formulating a problem in a particular model form (for an LP, a linear objective function and linear constraints).

Use of a technique can mean a wide range of possible model evaluation methods, with varying levels of sophistication. Similarly models associated with a particular evaluation technique may vary substantially in the detail incorporated. Thus a distinction can be made between simple and complex models, and between elementary and 'expert' use of model evaluation techniques. Surveys on the use made of techniques need to address these dimensions if they are to have any real value.

What surveys on the use of techniques do highlight is that very little work appears to have done on why 'techniques' are not more widely used. Apart from obvious practical difficulties in exploring this issue, there may also be a concern to avoid advocacy of a 'tool bag' approach to managerial problems. Accepted wisdom is that, rather than look for problems to which a particular tool can be applied, one should primarily seek to address important problems. This assumes that the OR practitioner is familiar with a wide variety of generic models and associated evaluation techniques and will apply these efficiently and effectively as a situation warrants. Increasingly this assumption is becoming unrealistic, as both techniques and associated software packages proliferate and increase in sophistication. One response is an increase in specialisation of individual management scientists or groups. Another response is to continue offering a general service, but one which emphasises a pragmatic approach biassed towards simplicity, robustness and transparency in model formulation, together with a willingness for qualitative as much as quantitative analysis. This is frequently what the client needs, at least to start with. However, while this pragmatic approach is useful, (and there is certainly extensive scope for it), opportunities for even more effective interventions, making greater use of quantitative techniques may be overlooked or ignored. Any number of explanations for this are possible, including the following:

1 A belief that standard models and associated techniques are not applicable in the kinds of problems typically addressed. Where problems are amenable to particular techniques these are handled by specialist groups.

2 Lack of awareness or familiarity with certain techniques; expertise forgotten through lack of use.

3 Formal detailed, quantitative analysis is not needed because the problem is well understood or not large enough. 
$4 \quad$ Uncertainty about the applicability of a given technique - are the associated assumptions valid in a given situation? What is the effect if they are not?

5 Lack of time to apply (sophisticated) techniques - client wants quick analysis/answers. Relevant techniques are too complicated (difficult) to apply in the time available and there is a need to avoid 'paralysis by analysis'.

6 Insufficient data available to use a given technique.

7 Difficult or impossible to quantify situation factors.

One or more of these perceptions may be appropriate in a given situation, and one may lead to another. For example, a perception that certain techniques are not often applicable is likely to lead to a loss of expertise and awareness of those techniques. Investment of time and effort in enhancing expertise in particular techniques may not be considered a cost effective option unless an application problem is very big or recurrent.

Successful interventions require modelling approaches which are simple, flexible, easily understood, appropriate to the situation, able to cope with low quality data, and able to help managers make decisions. With such arguments it is often easy to justify a simple, ad hoc approach, or at least one that involves only elementary application of a standard technique. Thus lack of expertise or awareness of useful techniques can be camouflaged by perceptions 4-7 above, and arguing that the client's needs will be met by something quick and dirty, or that using sophisticated techniques will make things more difficult to implement. In addition, models which are simple in terms of form and detail, that is 'constructively simple', tend to have a number of advantages over complex models. Broadly speaking, the advantages of constructively simple models can include some or all of the following:

- less time consuming and costly to develop;

- easier to modify;

- less demanding of data;

- assumptions are more transparent and accessible;

- sensitivity analysis is more practicable.

Notwithstanding the above arguments, competitive pressures ought to drive OR practitioners to make more sophisticated use of quantitative techniques, and more use of sophisticated techniques. In part there is a need for ongoing education of OR practitioners about new and existing techniques. But there is also a need for a much stronger applied emphasis in the OR literature. This should be a primary concern in the development of general models and associated evaluation procedures. application.

Ideally, general models and associated evaluation methods ought to lead directly to applications, but many practitioners might be forgiven for believing that this is not the case. Some might even argue that this is less likely to be the case when model development is pursued as a theoretical activity divorced from immediate problem application. A contributing factor to such perceptions is that theorists too often fail to consider adequately whether their models and analysis can be used in a practical context, if they are inclined to consider it at all.

If wide practical application is a concern, theorists and other developers of 'general' models must acknowledge the perceived advantages of constructively simple models, the need to demonstrate cost effectiveness, and points 4-7 above. When violations of model assumptions are likely, but difficult or impossible to assess, or when additional criteria and conditions are impossible to incorporate, the practical relevance of any model must be in question. The introduction of convenient, if esoteric, assumptions to make analytical solutions possible is not always helpful in an applied context, however elegant the results from a theoretical perspective. Furthermore, the values of model parameters are often rough estimates at best. This may undermine the value of refined analysis.

Faced with a choice about level of detail or model form, it can be useful to know how much better one model's solution may be from another. In particular, it is important to compare advice produced by different models for the full range of possible values of problem parameters. Analytical comparisons may be preferred, but failing this a large number of sample problem situations should be considered. OR practitioners need to be convinced that the extra effort (and communication problems) involved in more sophisticated modelling approaches will produce significantly superior recommendations to those of a simpler approach.

Possibly, ignorance or difficuity in comprehending particular techniques are reasons for the limited use made of some techniques in practice. But practitioners often have justifiable grounds for avoiding many theoretical models of decision problems based on a perceived failure of these models to exhibit sufficient 'applied' characteristics. Those interested in the wider diffusion of OR techniques need to bear this in mind.

\section{Stephen Ward}

